\title{
Monitoring the deformation of the metal aluminum film with glass substrate based on DSCM
}

\author{
Jie Han Xiaoou Pan Wenguang Xie Yan Li Zhongjing Li and \\ Qiang Wang \\ Department of physics, University of Heilongjiang \\ Email: wangqiangid@aliyun.com
}

\begin{abstract}
Keywords: metal aluminum film,glass substrate ,DSCM
Abstract. This work demonstrates the use of the digital speckle correlation method to analyse the deformation of the metal aluminum films with glass substrate under temperature field. Our results can be adapted to further extend the applications and provide important reference for the designing of the key coating parameters.
\end{abstract}

\section{Introduction}

With the development of the deep-space mission, space communication and earth observations technologies, space telescopes, radar, energy aggregator and other optical devices all have coating films structure for improved optical properties. Due to the strong ultraviolet light (thermal radiation) of the orbit environment, the exposed optical film of the high reflectivity mirror, antireflection mirror and other optical components easy to be cracked and off, the corresponding refractive index and film thickness also could be changed. Along with the using time increasing and outside space factors, the optical system performance maybe deteriorate, even made great economy loss. Meanwhile, the optical films are widely used in high- powered lasers systems. The thermal absorption of the films will lead to much large temperature gradient response on the whole optical film component, when the optical component temperature increased by laser illuminated. The great range thermal stresses would be occurred from difference of thermal expansion constants between the film and substrate and cause further film damage. The thermal effect is the critical factors that result in film damage[1-8].

The surface damage of optical films could further enhance the absorption and scatter of the film so that the part transmission and reflection will decreased. So it is very important to develop a structural health monitoring method to monitor the deformation and damage of the optical films under the temperature field. The traditional sensors and monitoring technologies are partly or totally failed as temperature effects. The optical measurement methods and technologies, especially noncontact optic methods are widely used in various environments, which could be realized in the whole field structure measurement. Because of the advantages of optic methods, such as strong antiinterference ability, high sensitivity, and brand using range, they have been great attention in recent years.

Digital Speckle Correlation Measurement (DSCM) based on digital image processing techniques has been the important method for deformation of materials and structures measurement, which apply to different scales measurement experiments and can be used for various fields [9-12]. DSCM demonstrate simple data processing method, high measurement sensitivity, simple operation process and reusability, except for the common whole field, non-contact and high precious measurements as other general optic methods. The objective of this work is to monitor the strain distribution and deformation process of the optic films under uniform temperature field based on DSCM.

\section{Theoretical Approach of DSCM}

Digital speckle correlation method (DSCM) is a kind of non-contact and full-field optical measurement method and widely applied in modern experimental mechanics, which can efficiently and feasibly analyze mechanical property of thin film material. The basic principle of DSCM is 
introduced as following: (1) Two images of the film's surfaces before and after deformation are captured and compared the difference; (2) The displacement field of film's surface could be measured using interrelation of probability statistics; (3) the calculation formula of deformation could be analyzed and the strain distribution of the film's surface could be calculated. The precision and calculated method of the correlation coefficient plays a key role in DSCM.
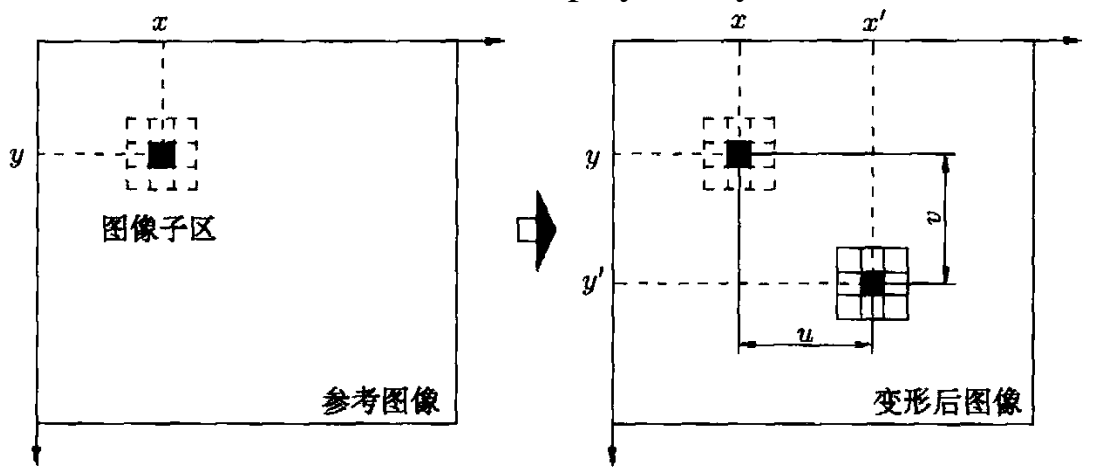

Fig. 1 Schematic diagram of 2-D Digital Image Correlation

The correlation coefficient is match of gray distribution for image sub-region, which could reflect regional similarity between before and after deformation images [11]. The coefficient is given by the expression.

$$
C(u, v)=\frac{\sum_{i=1}^{m} \sum_{j=1}^{m}\left[I_{1}(x, y)-\overline{I_{1}}\right]\left[I_{2}\left(x^{\prime}, y^{\prime}\right)-\overline{I_{2}}\right]}{\sqrt{\sum_{i=1}^{m} \sum_{j=1}^{m}\left[I_{1}(x, y)-\overline{I_{1}}\right]^{2}} \sqrt{\sum_{i=1}^{m} \sum_{j=1}^{m}\left[I_{2}\left(x^{\prime}, y^{\prime}\right)-\bar{I}_{2}\right]^{2}}}
$$

When $I_{1}(x, y)$ and $C(u, v)$ are the functions of gray for before and after deformation images, $\bar{I}_{1}$ and $\bar{I}_{2}$ are the average of $I_{1}(x, y)$ and $I_{2}(x, y), C(u, v)$ is correlation. The maximum value of Eq. (1) can be calculated by Newton-iterative method, and then the displacement of sub segment can be given, the strain is derived from by using displacement distribution at last.

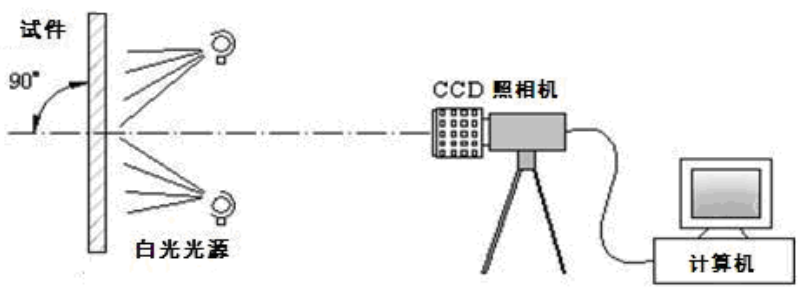

Fig .2 The picture of Experimental device for the 2D DIC method

\section{Results and Discussion}

The single layer aluminum reflection film samples on $\mathrm{K} 9$ glass substrates were obtained from Da Heng photoelectric company of Beijing, the thickness and diameter of the film are $5 \mathrm{um}$ and $15 \mathrm{~mm}$. Figure 2 depicts the experimental setup for the deformation measurements. PTC was used as heating source for obtained stable temperature field, the temperature distribution of the optic film was detected by infrared thermometer. For the aluminum film with four sides fixed, a CCD detector captured the change of the surface. Carbon toners sprayed into the surface of the aluminum film to forming the randomly distributed artificial speckle. When the temperature of aluminum film is increased, the temperature of the toner particles will also increase. In the experiment, the aluminum film and the artificial speckle are simplified considered as a intergrity and deform coordinated. The randomly distributed artificial speckle of the aluminum film surface is shown in Fig. 3. 

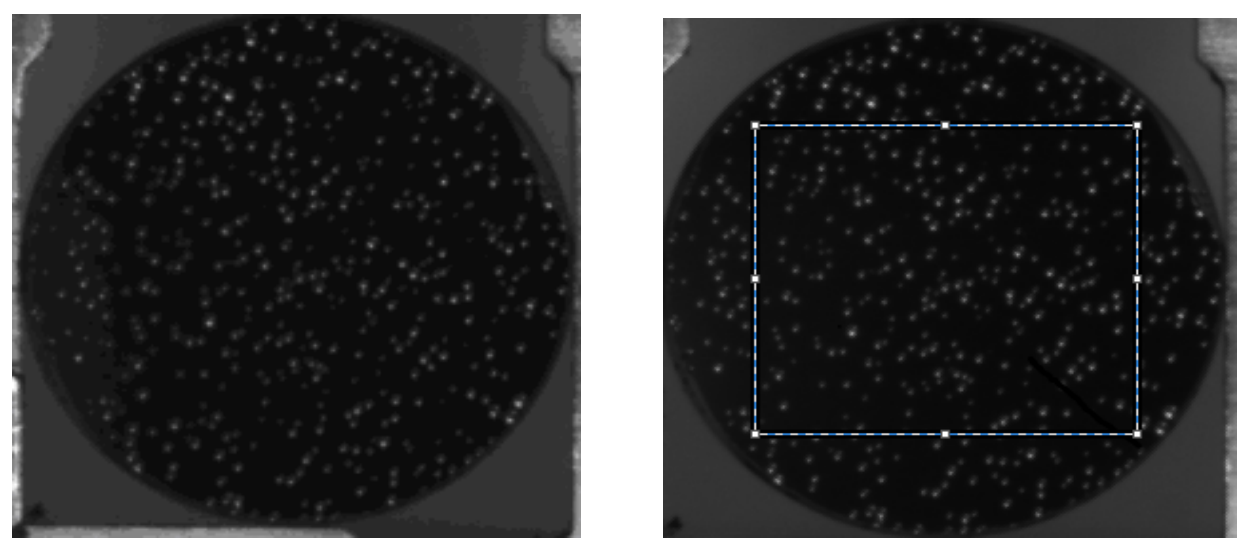

Figure 3. The tested specimen with artificial speckles and computational area Several aluminum films with artificial speckles were prepared. Firstly, the original image of the film at the room temperature $\left(25^{\circ} \mathrm{C}\right)$ was captured by using the $\mathrm{CCD}$. Secondly, at $52{ }^{\circ} \mathrm{C}, 77^{\circ} \mathrm{C}, 96$ ${ }^{\circ} \mathrm{C}$, the changed images of the film were captured. Two images before and after the deformation were attained and analyzed. From the high-quality surface displacement fields of the aluminum film obtained by VIC, surface strain distributions can be achieved.

The deformations of the aluminum film with temperature were obtained by multiple experiments. As Fig.4 and 5 shown, the stress distributed discrete and the partial stress is large from analyzing the deformation data of the $\mathrm{U}$ and $\mathrm{V}$ fields. The main reason is the residual stresses induced by lattice mismatch and other factors during the coating process. The stress distributions are nonuniformity that the strain distributions are non-uniformity and random. 


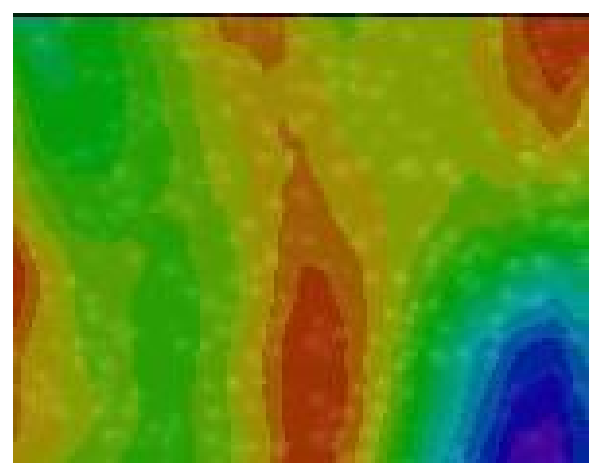

(a) $25{ }^{\circ} \mathrm{C}$

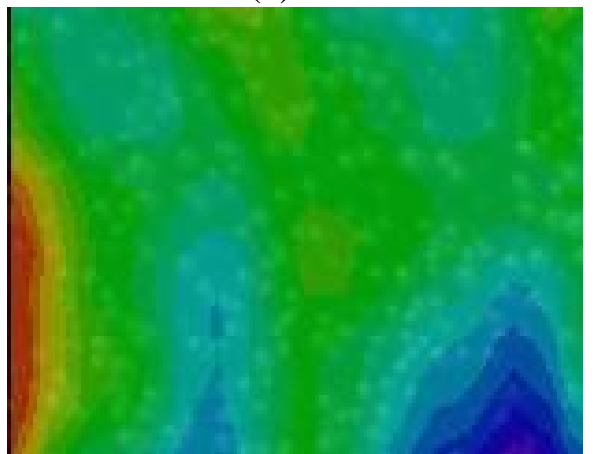

(c) $77^{\circ} \mathrm{C}$

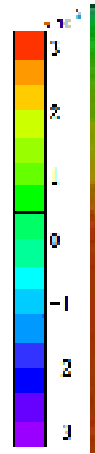

.

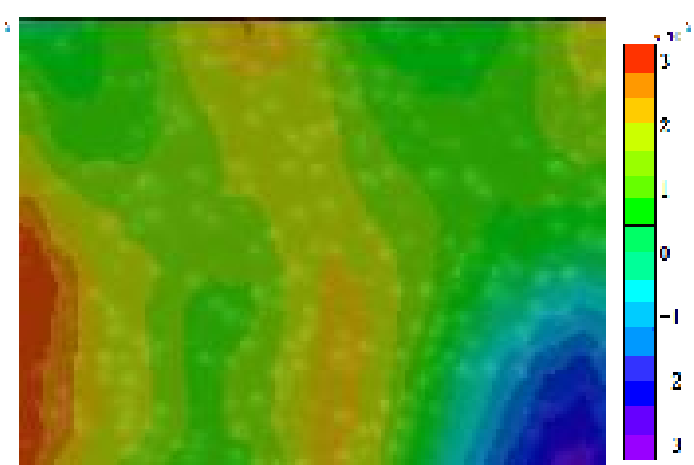

(b) $52{ }^{\circ} \mathrm{C}$
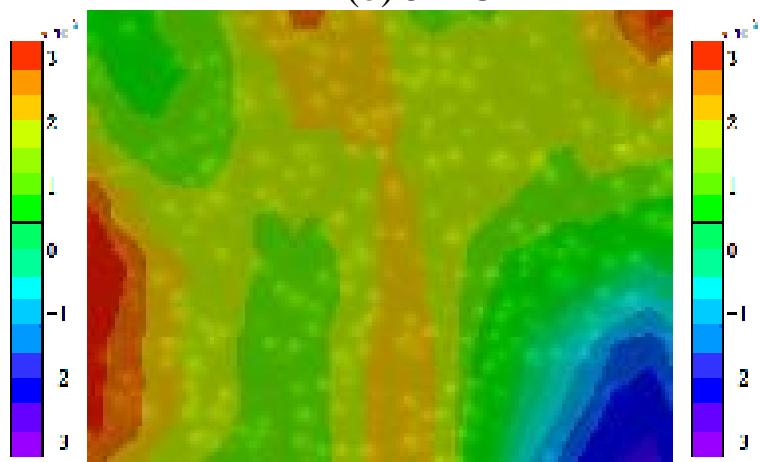

(d) $96{ }^{\circ} \mathrm{C}$

Figure $4 \mathrm{U}$ strain field for film surface structure changes with temperature

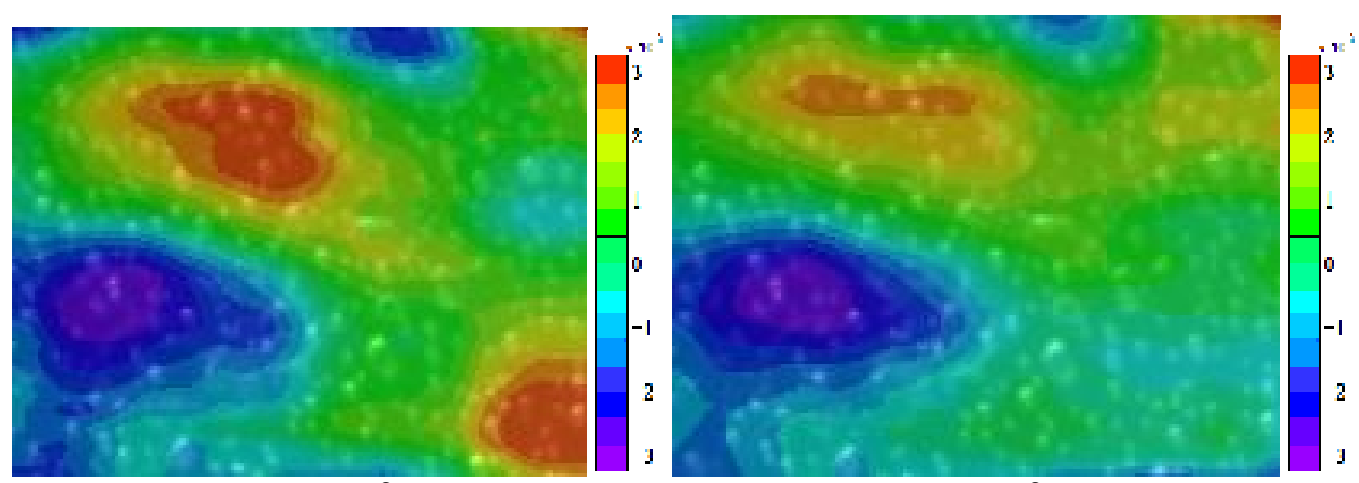

(a) $25^{\circ} \mathrm{C}$

(b) $52{ }^{\circ} \mathrm{C}$

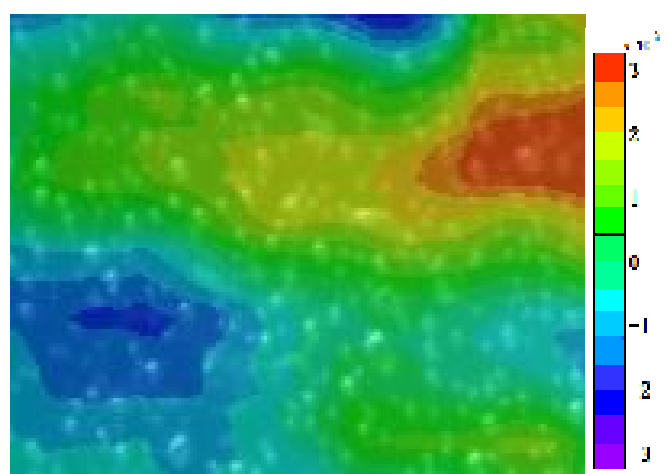

(c) $77^{\circ} \mathrm{C}$

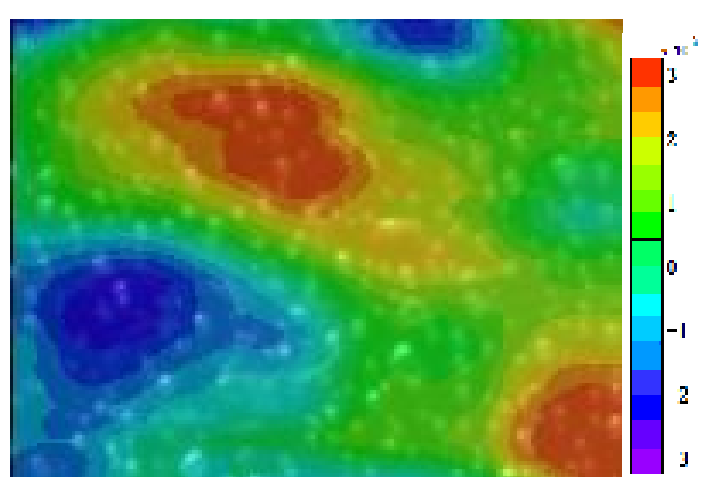

(d) $96{ }^{\circ} \mathrm{C}$

Figure 5. V strain field for film surface structure changes with temperature 


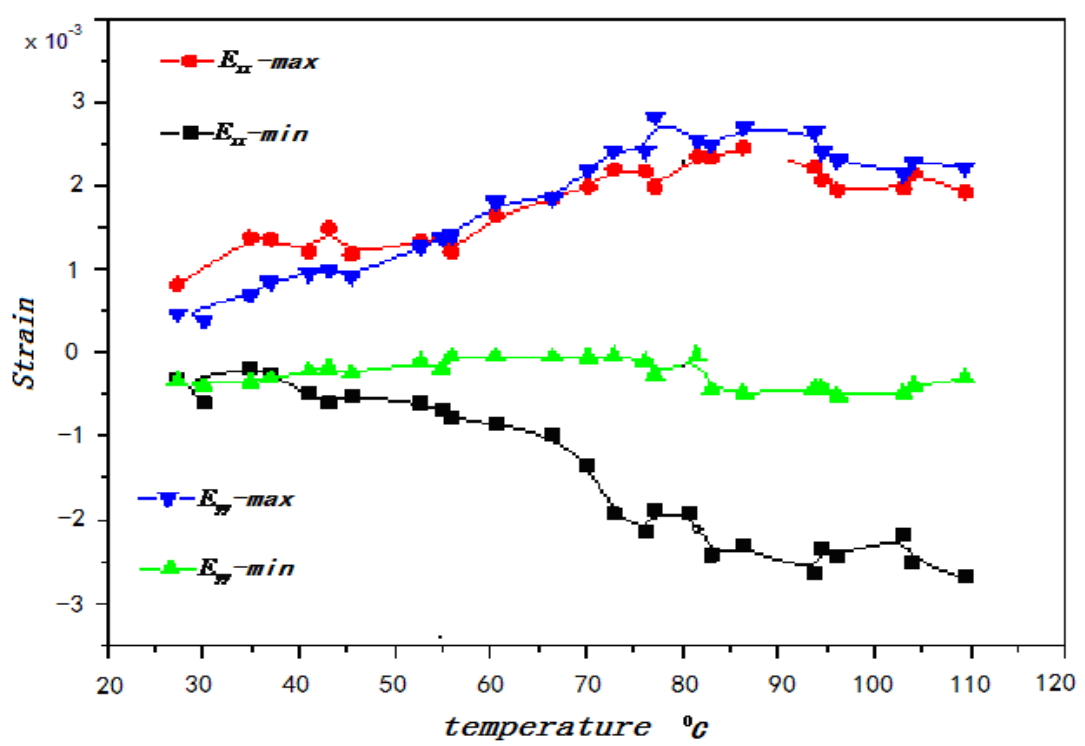

Figure 6. The strain with temperature changes

The strain of the local speckles with initial high strain decreases or increases as temperature rising. The strain variation is no single direction. The obvious change of the strain occurs at $80 \mathrm{oC}$ in the same computational area from the $\mathrm{U}$ and $\mathrm{V}$ strain distributions in the figure, due to the residual stress releasing during the heating process. At first, the deformation of the film surface mixes the residual deformation and thermal deformation. And then, temperature rising benefits residual stress release, the thermal deformation can be investigated obviously. The strain distributions of $\mathrm{U}$ and $\mathrm{V}$ field are relatively stable at $80 \mathrm{oC}$. When the temperature rises above $100 \mathrm{oC}$, the film can suffer damage, even to strip at last.

The maximum strain and minimum strain of the $\mathrm{U}$ and $\mathrm{V}$ fields with temperature changed are showed in Fig. 6. The same result can be gained in numeral repeated tests, which can be reflected the temperature influence factors with the film deformation. The maximum strain of the aluminum film is $0.25 \%-0.28 \%$. The compress strain of $\mathrm{V}$ field is not changed obviously. The reasons and mechanism should still be further discussed and analyzed.

\section{Conclusion}

In this investigation, a simple and practical scheme for the surface deformation of the aluminum film with temperature has been demonstrated based on DSCM. The thermal deformation of the single layer aluminum reflection film samples under various changed temperature were investigated. The experimental results can be effectively studied the thermal damage and mechanism, and eliminated the residual strain using the thermal load during the coating process. Related conclusions can provide important reference for designing the coating technology.(This Project is supported by the education department of Heilongjiang 11531277).

\section{References}

[1] Pan Yingjun, Feng Jun. High-power laser damage to optical films. Semiconductor Optoelectronics, 1997, 18(1): 61.65.

[2] Du Yanyi, An Jianzhu, Shu Xiaojian. Effect of thermal distortion of mirror on characteristics of laser beam in unstable resonator. High Power Laser and Particle Beams,2008,20(8): 1333-1338).

[3] Zhou Renzhong. Adaptive optics. Beijing: National Defense Industry Press, 1996.

[4] Neal D R, Copland J, Neal D. Shack-Hartmann wavefront sensor precision and accuracy[C]//Proc of SPIE. 2002,4779: 148-160.

[5] S Ri. Thermal fatigue of high-purity aluminum thin films under thermal cycle testing [J] .Strength, Fracture andComplexity, 2011 (7) : 61-70.

[6] K Jang. Effect of Capping Layer on Hillock Formation in Thin Al Films [J]. Metals and 
Materials International. 2008 (14) : 147-150.

[7] M Saka, R Nakanishi. Fabrication of Al thin wire by uti- lizing controlled accumulation of atoms due to electromi- gration [J]. Materials Letters, 2006 (60) : 2129-2131.

[8] Sun Xilian, Fan Zhengxiu, Shao Jianda. Effects of Al2O3 and $\mathrm{Cr}$ interlayers on the optical property and adhesion of Ag film. Acta Metallurgica Sinica, 2007, 43(3):307-310.

[9] Su Y, Zhang Q, Gao Z, et al. Noise-induced bias for convolution-based interpolation in digital image correlation.[J]. Optics Express, 2016, 24(2).

[10] Ronghua Z, Huimin X, Zhenxing H, et al. Performances of different subset shapes and control points in subset-based digital image correlation and their applications in boundary deformation measurement.[J]. Applied Optics, 2015, 54(6):1290-301.

[11] RUAN Le-le, WANG Wei-ze, Effect of Imaging Mode of Scanning Electron Microscope on the results of Digital Speckle Correlation Method. Journal of East China University of Science and Technology[J] .Vol. 41No. 32015 (6) : 412-416.

[12] Pan B. Bias error reduction of digital image correlation using Gaussian pre-filtering[J]. Optics \& Lasers in Engineering, 2013, 51(10):1161-1167. 